\title{
A Tale From the Early Stone Age: Pediatric Ureterolithiasis as Appendicitis Mimic - A Case Report and Management Overview
}

\author{
Neil P. Larson ${ }^{1}$, Rachel E. Bridwell ${ }^{1}$, Michael J. Yoo ${ }^{1}$ \\ 1. Emergency Medicine, Brooke Army Medical Center, Fort Sam Houston, USA
}

Corresponding author: Neil P. Larson, neilplarson@gmail.com

\begin{abstract}
Ureterolithiasis in the pediatric population is uncommon and may mimic acute abdomen. While the majority of small stones pass spontaneously, complications may ultimately warrant surgical intervention. As the majority of affected patients have a predisposing condition, targeted therapy with close and consistent follow-up may prevent recurrence, emphasizing timely diagnosis. The authors present the case of a previously healthy 13 -year-old boy with eight days of right lower quadrant abdominal pain and emesis, who was found to have a distal ureteral stone necessitating surgical intervention.
\end{abstract}

Categories: Emergency Medicine, Pediatrics, Urology

Keywords: ureterolithiasis, pediatric urinary stone disease

\section{Introduction}

Unlike in the adult population, pediatric urinary stone disease is an uncommon emergency department (ED) diagnosis. Patients less than 18 years of age comprise only $2 \%-3 \%$ of the urinary stone patient population, with the estimated incidence of pediatric urinary stone disease at approximately 59.5 cases per 100,000 person years $[1,2]$. Incidence increases with age peaking in adolescence, and there is a slight female predominance $[2,3]$. Caucasians consist of the vast majority $(88 \%)$ of pediatric urinary stone patients $[2,4]$. Flank or abdominal pain is the most common presenting symptom followed by gross hematuria $[4,5]$.

Review began 09/16/2020 Review ended 09/23/2020 Published 09/24/2020

() Copyright 2020 Larson et al. This is an open access article distributed under the terms of the Creative Commons Attribution License CC-BY 4.0., which permits unrestricted use, distribution, and reproduction in any medium, provided the original author and source are credited.

\section{Case Presentation}

A 13-year-old previously healthy African American male presented to the ED for eight-day duration of right lower quadrant pain and new onset intractable nausea and vomiting. He was previously evaluated at another facility four days prior and discharged home with symptomatic therapy. On review of systems, the patient denied recent travel, fever, abdominal trauma, previous abdominal surgeries, blood dyscrasias, or diarrhea. The patient's initial vital signs were blood pressure of $93 / 61 \mathrm{~mm} \mathrm{Hg}$, heart rate of 123 beats per minute, respiratory rate of 20 breaths per minute, and oxygen saturation of $98 \%$ in room air with a temperature of 98.7 degrees Fahrenheit. Physical exam was notable for mild right lower quadrant tenderness with rebound, but negative for costovertebral angle tenderness or testicular tenderness. Laboratory analysis reviewed a white blood cell count of 15,200 cells/ $\mu \mathrm{L}$ with an $85 \%$ neutrophilic predominance, though comprehensive metabolic panel and lipase were unremarkable. Specifically, the patient's blood urea nitrogen (BUN) and creatinine were $17.9 \mathrm{mg} / \mathrm{dL}$ and $0.93 \mathrm{mg} / \mathrm{dL}$, respectively, and the urinalysis was without blood, nitrite, or leukocyte esterase, but did contain 1+ ketones and a mildly elevated specific gravity of 1.031 (normal range 1.005-1.025). The patient underwent evaluation for acute appendicitis, and while the abdominal ultrasound (US) was non-diagnostic, MRI demonstrated a normal appendix, right perinephric fat stranding, mild hydronephrosis, and a 3 millimeter $(\mathrm{mm})$ ureterolith at the right ureterovesicular junction (Figure 1). The patient received 1,000 mL of Ringer's lactate solution, $650 \mathrm{mg}$ of intravenous acetaminophen, and $4 \mathrm{mg}$ of intravenous ondansetron, with resolution of tachycardia and improvement of symptoms. Urology was consulted for further management, and the patient was admitted to the hospital for trial of medical therapy including medical expulsive therapy. However, due to refractory pain and emesis without stone passage, coupled with possible ureteral obstruction, the patient subsequently underwent successful cystoscopy, ureteroscopy, laser lithotripsy, and ureteral stent placement. The patient was discharged after an uneventful postoperative course, and noted resolution of symptoms at postoperative follow-up appointment. 


\section{Cureus}

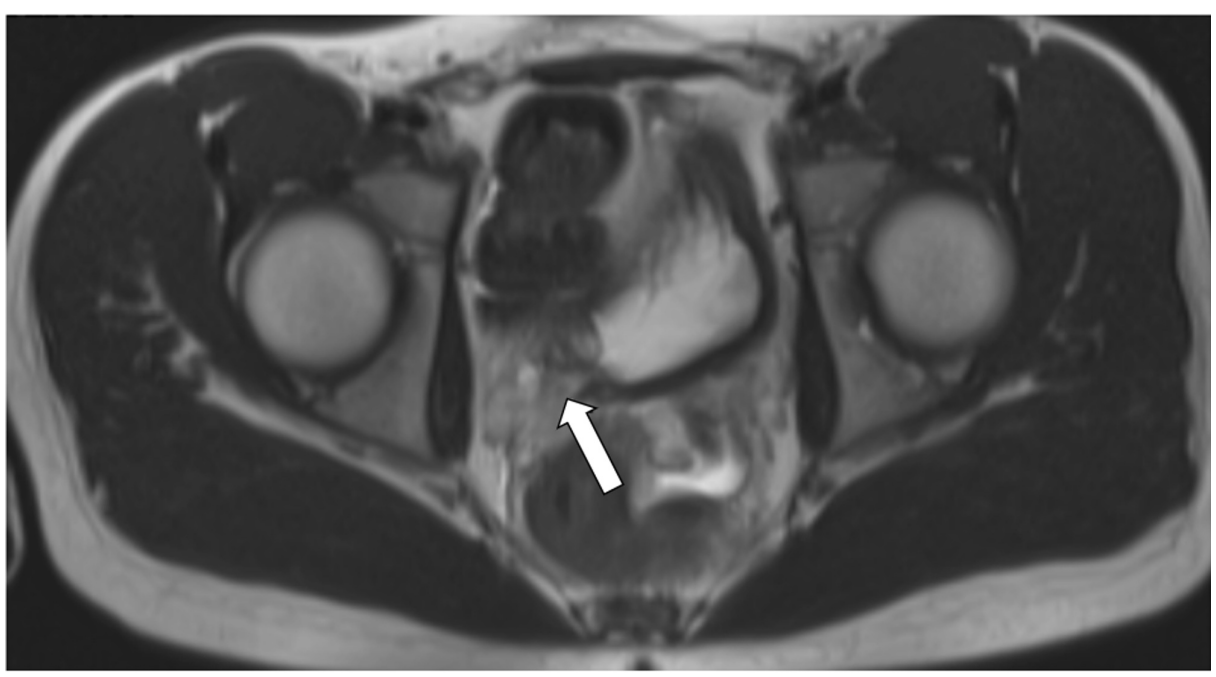

FIGURE 1: Axial view of HASTE pelvic MRI demonstrating approximately three millimeter ureteral stone near the right ureterovesicular junction with mild ureteral dilatation (white arrow).

HASTE, Half-Fourier acquisition single-shot turbo spin echo

\section{Discussion}

The differential diagnosis of right lower quadrant abdominal pain in the pediatric population is broad and includes appendicitis, testicular or ovarian torsion, infection, and referred musculoskeletal pain from the lower extremities. Although uncommon, ureterolithiasis may present similar to an acute surgical abdomen, with patients exhibiting acute onset of nausea and vomiting, intractable pain, and peritoneal signs. Workup should begin with a thorough physical exam, including evaluation of the back, abdomen, hip, and pelvis, potential hernia sites, and genitals as necessary. While non-specific, new onset gross or microscopic hematuria on urinalysis supports the diagnosis of a kidney stone [6]. Additionally, a urinalysis and urine culture should be obtained to rule out a concomitant infection. A renal function panel is essential to evaluate overall renal performance. Ultimately, imaging is needed to confirm a suspected diagnosis of acute urinary stone. Although commonly ordered due to high sensitivity and specificity (up to $98 \%$ and $99 \%$, respectively), CT of the abdomen and pelvis should be avoided, if possible, in pediatric patients due to radiation exposure and significantly higher study cost compared to US. As pediatric body habitus is generally more amenable to US, with a reported sensitivity and specificity of $76 \%$ and $100 \%$, renal US remains the initial preferred imaging modality in evaluating for pediatric urinary stones. However, as with our patient, smaller stones and distal ureteral stones are more likely to be missed on US [7]. With an estimated sensitivity and specificity of $82 \%$ and $98 \%$, MRI can also be used as a radiation sparing modality [8]. However, due to duration of study, which may potentially require sedation, and significantly increased cost in comparison to both US and CT, MRI only accounts for approximately $2 \%$ of imaging in pediatric urinary stone patients, and is more difficult to obtain in the $\mathrm{ED}[8,9]$. As such, initiating imaging workup for suspected acute urinary stone with renal US in the pediatric population is the recommendation of the authors as well.

The initial treatment of pediatric acute urinary stone disease should include aggressive hydration, antiemetics for nausea, and analgesia. According to recent American Urological Association (AUA) guidelines, ureter stone sizes of less than or equal to $10 \mathrm{~mm}$ in pediatric patients may be observed and trialed with medical therapy [10]. While other studies and hospital guidelines may vary according to recommended management of stones based on size alone, a recent study in accordance with AUA guidelines suggested that stones less than $6.7 \mathrm{~mm}$ in size have greater than an $80 \%$ chance of spontaneous passage [11]. Subsequently, a hemodynamically stable patient with a small, uncomplicated stone with well-controlled pain and nausea can be discharged with close urology follow-up. Barring contraindications, outpatient symptomatic therapy includes non-steroidal anti-inflammatory drugs (NSAIDs), acetaminophen, opioid analgesia for breakthrough pain, medical expulsive therapy, and a urine strainer for stone capture. However, underlying renal disease, solitary kidney state, larger stone size, complete ureter obstruction, concomitant urinary tract infection, and struvite stones necessitate urological evaluation for surgical intervention due to an increased risk for inability to pass stone, acute decompensation, and long-term morbidity [10,12-15]. Intractable pain and failure of stone passage with trial of medical therapy, as experienced by our patient, similarly merit urology evaluation $[4,10]$. Similar to the adult population, calcium oxalate stones are the most common, followed by calcium phosphate stones trailed by smaller portions of several other types [16]. Although any pediatric patient can develop stone disease, the majority of pediatric stone patients have underlying predisposing conditions, including metabolic abnormalities (hypercalciuria, hyperoxaluria, 
among several others), genitourinary tract structural abnormalities, and urinary tract infections $[3,5,16]$. Unfortunately, as the majority of pediatric patients experience recurrent episodes of stones, timely diagnosis and tailored treatment targeting the underlying predisposition should be aggressively pursued in conjunction with urology.

\section{Conclusions}

Pediatric urinary stone disease is an uncommon diagnosis and may mimic more common causes of acute abdomen to include appendicitis. In addition to a thorough physical examination, laboratory workup including urinalysis and imaging to include renal US when possible will aid in diagnosis. While hydration, analgesia, and antiemetics are the mainstay of treatments and most stones pass spontaneously, emergent urology consultation for procedural intervention is warranted in multiple circumstances to prevent complications. Predisposing conditions including metabolic abnormalities are present in the majority of patients and warrant follow-up and therapy targeted at stone recurrence prevention when possible.

\section{Additional Information \\ Disclosures}

Human subjects: Consent was obtained by all participants in this study. Conflicts of interest: In compliance with the ICMJE uniform disclosure form, all authors declare the following: Payment/services info: All authors have declared that no financial support was received from any organization for the submitted work. Financial relationships: All authors have declared that they have no financial relationships at present or within the previous three years with any organizations that might have an interest in the submitted work. Other relationships: All authors have declared that there are no other relationships or activities that could appear to have influenced the submitted work.

\section{Acknowledgements}

The view(s) expressed herein are those of the author(s) and do not reflect the official policy or position of Brooke Army Medical Center, the U.S. Army Medical Department, the U.S. Army Office of the Surgeon General, the Department of the Army, the Department of the Air Force and Department of Defense or the U.S. Government.

\section{References}

1. Schwarz RD, Dwyer NT: Pediatric kidney stones: long-term outcomes. Urology. 2006, 67:812-816. 10.1016/j.urology.2005.10.020

2. Ward JB, Feinstein L, Pierce C, et al.: Pediatric urinary stone disease in the United States: the urologic diseases in America project. Urology. 2019, 129:180-187. 10.1016/j.urology.2019.04.012

3. Bowen DK, Tasian GE: Pediatric stone disease. Urol Clin North Am. 2018, 45:539-550. 10.1016/j.ucl.2018.06.002

4. Bush NC, Xu L, Brown BJ, et al.: Hospitalizations for pediatric stone disease in United States, 2002-2007 . J Urol. 2010, 183:1151-1156. 10.1016/j.juro.2009.11.057

5. Gearhart JP, Herzberg GZ, Jeffs RD: Childhood urolithiasis: experiences and advances. Pediatrics. 1991, 87:445-450.

6. Kit LC, Filler G, Pike J, Leonard MP: Pediatric urolithiasis: experience at a tertiary care pediatric hospital . Can Urol Assoc J. 2008, 2:381-386. 10.5489/cuaj.804

7. Passerotti C, Chow JS, Silva A, et al.: Ultrasound versus computerized tomography for evaluating urolithiasis. J Urol. 2009, 182:1829-1834. 10.1016/j.juro.2009.03.072

8. Brisbane W, Bailey MR, Sorensen MD: An overview of kidney stone imaging techniques . Nat Rev Urol. 2016, 13:654-662. 10.1038/nrurol.2016.154

9. Routh JC, Graham DA, Nelson CP: Trends in imaging and surgical management of pediatric urolithiasis at American pediatric hospitals. J Urol. 2010, 184:1816-1822. 10.1016/j.juro.2010.03.117

10. Surgical management of stones: AUA/Endourology Society Guideline (2016) . (2016). Accessed: September 14, 2020: https://www.auanet.org/guidelines/kidney-stones-surgical-management-guideline.

11. Elmacı AM, Dönmez Mİ, Akın F, Çetin B, Gündüz M: What predicts spontaneous passage of $\leqslant 1 \mathrm{~cm}$ ureteral stones in children?. J Pediatr Surg. 2020, 55:1373-1376. 10.1016/j.jpedsurg.2019.05.012

12. Parvex P, Pippi-Salle JL, Goodyer PR: Rapid loss of renal parenchyma after acute obstruction . Pediatr Nephrol. 2001, 16:1076-1079. 10.1007/s004670100031

13. Gupta M, Bolton DM, Gupta PN, Stoller ML: Improved renal function following aggressive treatment of urolithiasis and concurrent mild to moderate renal insufficiency. J Urol. 1994, 152:1086-1090. 10.1016/S0022-5347(17)32509-0

14. Silverman DE, Stamey TA: Management of infection stones: the Stanford experience. Medicine. 1983, 62:44-51. 10.1097/00005792-198301000-00004

15. Jun E, Metcalfe P, Mandhane PJ, Alexander RT: Patient and stone characteristics associated with surgical intervention in pediatrics. Can J Kidney Health Dis. 2015, 2:1-6. 10.1186/s40697-015-0057-6

16. Milliner DS, Murphy ME: Urolithiasis in pediatric patients. Mayo Clin Proc. 1993, 68:241-248. 10.1016/S0025-6196(12)60043-3 